Reprod. Nutr. Dévelop., 1987, 27 (1 B), 205-206.

\title{
Activités alimentaires et niveau d'ingestion chez la jument gestante et allaitante
}

Syiviane BOULOT, J. P. BRUN (*), M. DOREAU, W. MARTIN-ROSSET (**)

Laboratoire de la lactation,

( $\left.{ }^{*}\right)$ Atelier Electronique du Département Elevage et Nutrition des Herbivores,

(**) Unité Elevage et alimentation du cheval,

I.N.R.A. Theix, 63122 Ceyrat, France

Summary. A $65 \%$ increase in forage intake in lactating mares as compared with pregnant ones was associated with 15 and $24 \%$ increases in eating and chewing times, respectively. The rate of eating increased by $50 \%$ and was positively related to intake. All the mares showed a cyclic pattern of feeding activities.

Le comportement alimentaire du cheval peut être influencé par des variations modérées du niveau alimentaire (Doreau, 1978). Cependant, la nature et l'ampleur des modifications comportementales permettant aux juments d'accroître fortement leur niveau d'ingestion en fonction de leurs besoins ne sont pas connues; cet essai vise à les préciser.

Matériel et méthodes. Onze juments adultes de races Anglo-arabe et Selle français, d'un poids moyen de $530 \mathrm{~kg}$, ont été maintenues en stalles individuelles en gestation, ou en boxes contigus en lactation. Elles ont reçu un régime de com: position constante à base de foin de prairie naturelle $19 \%$ de matières azotées totales, $30 \%$ de cellulose brute) offert à volonté en 2 distributions égales (à $8 \mathrm{~h} 30$ et à $16 \mathrm{~h}$ ), complété par un aliment concentré ( $50 \%$ d'orge, $45 \%$ de tourteau de soja) apporté en une seule fois à 8 heures, à raison de $10 \%$ de la matière sèche totale ingérée. Leurs activités alimentaires ont été étudiées au cours du 9e mois de gestation et pendant le premier mois de lactation grâce à un appareil électronique portatif enregistrant les mouvements des mâchoires (Brun et al,, 1984). La durée d'ingestion a été calculée comme étant la somme des durées des repas, ceux-ci correspondant à des séquences d'activités masticatoires durant au moins $10 \mathrm{~min}$ et ne comprenant pas d'interruptions supérieures à $10 \mathrm{~min}$. Selon que la durée de mastication (durée d'ingestion - durée des interruptions), représentait plus ou moins de la moitié de la durée d'ingestion, l'ingestion a été qualifiée d'intense ou de lente, le rapport mastication/ingestion caractérisant son intensité. Le nombre de jours d'observations par animal ayant varié entre 1 et 8 et

TABL. 1. - Effet de l'état physiologique sur le comportement alimentaire des juments (moyennes \pm écart-type et nombre d'observations). D : différences entre stades significatives au seuil $1 \%\left(1^{* *}\right)$, $5 \%(*)$.

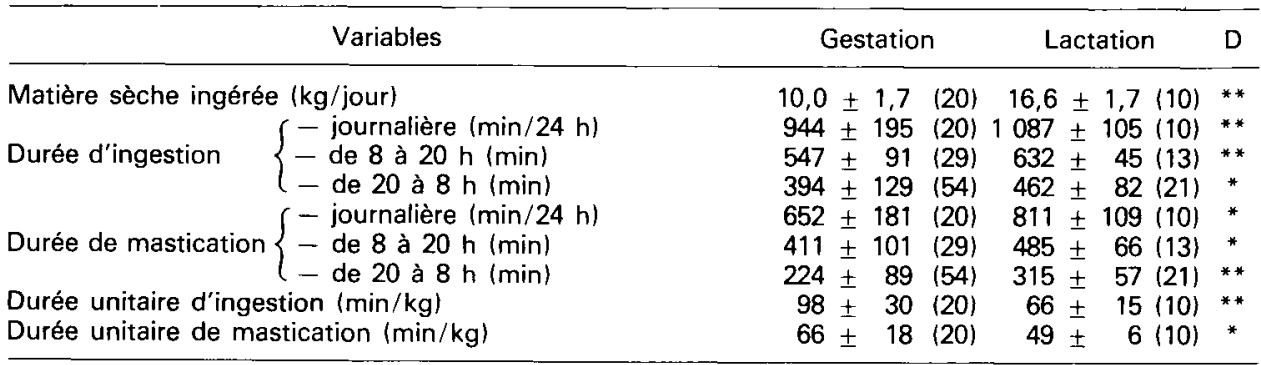


les enregistrements de 24 h obtenus étant peu nombreux, en particulier en lactation (tabl. 1), l'amplitude des variations journalières et individuelles n'a pas été déterminée. Les activités alimentaires ont également été étudiées sur des périodes de $12 \mathrm{~h}$; le nombre d'enregistrements pris en compte est alors plus important.

Résultats et discussion. Les quantités de matière sèche ingérées par les juments, voisines de $10 \mathrm{~kg} / \mathrm{j}$ au $9^{e}$ mois de gestation $(1,6 \%$ du poids vif), ont fortement augmenté pendant le premier mois de lactation, en moyenne de $65 \%$ par rapport à la gestation. Le nombre (11 en moyenne) et la répartition horaire des repas n'ont pas varié d'un état physiologique à l'autre ; les activités alimentaires ont présenté la même répartition cyclique au cours du nycthémère (fig. 1). Les durées journalières d'ingestion et de mastication, égales à 15 et $11 \mathrm{~h}$ en gestation, se sont accrues en lactation respectivement de 15 et $24 \%$ (tabl. 1). La plus grande dispersion des valeurs en gestation et pour des niveaux d'ingestion modérés pourrait être explicable par l'apparition fréquente chez les équidés à l'attache d'activités masticatoires non spécifiquement alimentaires, telles que le grignotage répété des bat-flancs ou de l'aliment, le trempage du fourrage dans l'eau de boisson (Willard et al., 1977). Quel que soit l'état physiologique, environ $40 \%$ de la durée journalière d'ingestion a eu lieu entre 20 et $8 \mathrm{~h}$. L'intensité de cette ingestion nocturne s'est accrue de $19 \%$ en lactation. Toutes données confondues, la durée journalière d'ingestion n'a pas été reliée aux niveaux d'ingestion, la durée journalière de mastication l'étant de façon peu étroite $(r=0,50 ; P<0,01 ; n=30)$. Par contre, la durée unitaire d'ingestion (durée d'ingestion/matière sèche ingérée), réduite en lactation, a été négativement reliée aux quantités ingérées aux deux stades physiologiques $(r=-0,73 ; P<0,01 ; n=30)$. L'intensité de l'ingestion a augmenté lorsque la durée unitaire d'ingestion diminuait, la relation entre ces deux variables étant cependant peu étroite. La durée unitaire de mastication a diminué plus faiblement lorsque le niveau d'ingestion augmentait.
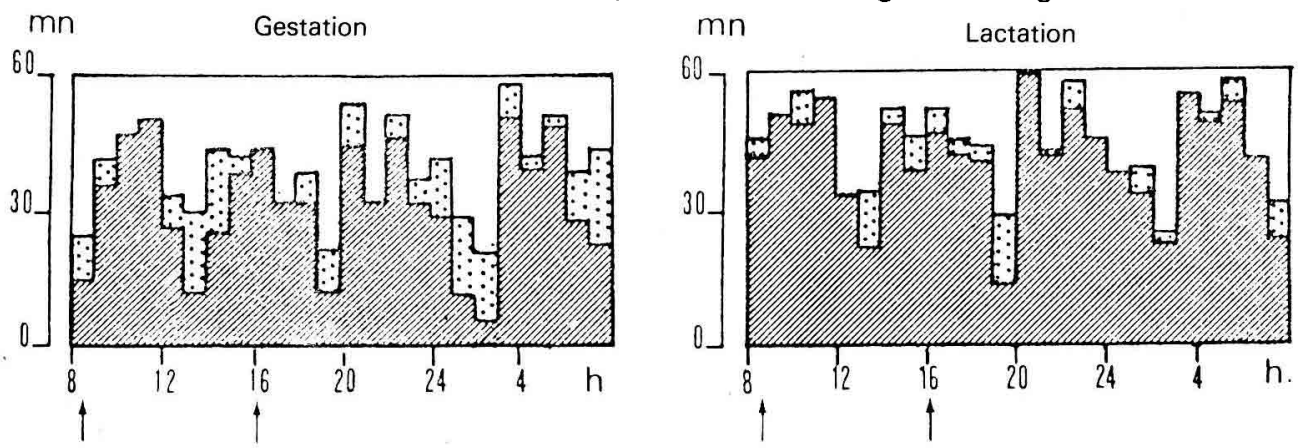

FIG. 1. - Répartition horaire des activités alimentaires des juments, $\$$ ingestion intense ; $:$ ingestion lente ; $\uparrow$ distribution du foin.

C'est donc plus par l'augmentation de la vitesse d'ingestion que par celle de la durée d'ingestion que les juments ont accru leurs consommations en lactation. La mesure de la valeur pondérale des prises alimentaires pourrait permettre de distinguer à l'avenir les activités alimentaires des autres activités masticatoires.

Brun J. P., Prache S., Béchet G., 1984. 5th Meet. Europ. Grazing Workshop, Edinburgh. Doreau M., 1978. Ann. Zootech., 27, 291-302.

Willard J. G., Willard J. C., Wolfram S. A., Baker J. P., 1977. J. anim. Sci., 45, 87-93. 